\title{
Localization in fractal spaces: Exact results on the Sierpinski gasket
}

\author{
Xiang Rong Wang \\ Department of Physics, Hong Kong University of Science and Technology, Clear Water Bay, Kowloon, Hong Kong
}

(Received 8 November 1994; revised manuscript received 15 December 1994)

\begin{abstract}
Localization due to space structure, rather than due to randomness, is investigated by studying the usual tight-binding model on the Sierpinski gasket. Some exact results are obtained from the decimation-renormalization-group method. It is surprising that there exist an infinite number of extended states on the Sierpinski gasket. This set of extended states forms a Cantor set. The rest of the states are exponentially localized except for two states that are localized in a power-law fashion. It can be shown that exponential localization of lengths of states on the Sierpinski gasket reveal a self-similar pattern.
\end{abstract}

Localization due to randomness is not surprising. However, localization can also be induced by space structure such as a fractal. In general, the electronic structure of a given system is determined by the effective potential on electrons and space. It is well known that crystalline materials can only admit Bloch waves due to their translational symmetries. In real life, structures of materials vary dramatically, ranging from crystallines to completely disordered structures. Single crystals are examples of the former, and porous media exemplify the latter. In 1984, Schechtman et al. ${ }^{1}$ discovered quasicrystals. The structure of a quasicrystal is something between a perfect crystal and a completely disordered system. Therefore it is interesting to study how particle states deviate from Bloch waves in those materials that are neither crystals nor completely disordered systems.

The mechanisms leading to the localization of electronic states are already known. In fact, in his seminal paper in $1958,{ }^{2}$ Anderson pointed out that electronic wave functions in a random potential may be profoundly altered if the randomness is sufficiently strong. He demonstrated, by using a simplified theoretical model, that a wave function may become localized when the disorder, due to the presence of impurities or imperfections in the lattice, is strong. Generally speaking, in a quantum system, there are both tunneling and interference effects within a wave function scattered by a random potential. These two aspects can affect each other and produce rich physics. Delocalized states, such as the Bloch states in perfect crystals, appear when the tunneling of particles (with certain energies) is enhanced by a constructive interference of these waves. On the other hand, when the interference is destructive, it may lead to a localization of the state. For example, phases of electron waves in disordered systems will be randomized due to scattering by impurities. The interference of the scattered waves is destructive if the disorder is strong enough. This leads to localization in disordered systems. However, it is very difficult to quantitatively (sometimes even qualitatively) determine whether an electronic state is localized or extended (delocalized) for a given disordered system. Tholess and coworkers ${ }^{3}$ formulated a scaling description for the localization problem. Abrahams et al. ${ }^{4}$ proposed a one-parameter scaling theory. In the theory, all electronic states of a noninteraction disordered system are localized when the dimension of a system is lower than or equal to 2 [two-dimensional (2D) disordered electron systems in a magnetic field have many nontrivial properties $^{5}$ ], and a mobility edge may exist for $d=3$.

In the past decade, major efforts have been directed to investigate wave-function properties of quasicrystalline systems due to the advances of technology and their possible applications. ${ }^{6,7}$ In particular, the electronic structure of one-dimensional quasicrystals have received, both theoretically ${ }^{8-12}$ and experimentally, ${ }^{6,7}$ the most attention for both its simplicity and experimental realization in superlattices. ${ }^{6}$ Localized, critical, and extended states have been reported to exist and coexist in some 1D quasicrystal models, such as quasiperiodic incommensurate modulated potentials ${ }^{8}$ and Fibonacci quasicrystals. ${ }^{10,11}$ Although some analytical approaches have been invented to study the electronic structure of these quasicrystal systems, there is still lack of an exact solution which can give us indisputable answers to the localization problem in quasicrystals. Our current understandings are confined to those obtained from numerical studies. ${ }^{8-12}$

Here, I present some exact results of localization in fractal spaces. The usual tight-binding model, with only the nearest-neighbor hopping considered, is used to describe a noninteracting particle system for simplicity. The electronic structure on the Sierpinski gasket is studied for several reasons. First, a fractal is a good description of porous media at small length scales because both have scale invariance structures. Similar to quasicrystals, deterministic fractals do not have a periodic structure like perfect crystals, but they do have certain long-range correlations. It will be interesting to study localization properties in such systems. A better understanding of localization in fractal spaces may shed light on the localization in quasicrystals and random systems in general. The more important reason is that we can solve the problem exactly. Domany et al. ${ }^{13}$ studied the density of states (DOS) of the Sierpinski gasket by using the recursion relation method. They found that the energy levels are discrete and degenerate, and the density of states per site approaches a limit with an infinite number of different kinds of singularities. They also conjectured that all states are localized. In order to investigate the localization properties of the tight-binding models on the Sierpinski gasket, I will use the decimation-renormalization method to derive the recursion relation of the hopping coefficient for a chosen energy state. Then the flow dia- 
gram can be obtained for the hopping coefficient, which contains information about wave functions of the system. ${ }^{12,14}$ Some surprising results are found. There exists an infinite number of extended states on the Sierpinski gasket. This set of extended states forms a Cantor set. Furthermore, it can be shown that exponential localization lengths of states on the Sierpinski gasket reveal a self-similar pattern. The rest of the states (including those gap states) are exponentially localized except two states which are localized in a power-law fashion.

To illustrate the method and its potential applications, let us first study the one-dimensional tight-binding model,

$$
\mathscr{H}=\sum_{\langle i j\rangle} t c_{i}^{\dagger} c_{j}
$$

where $\langle i j\rangle$ denote the nearest-neighbor pairs, $c_{i}^{\dagger}$ and $c_{i}$ are creation and annihilation operators, respectively, and $t$ is the hopping coefficient. It is well known that energy levels are given by $\varepsilon(k)=2 t \cos (k a)$, where $a$ is the lattice constant and $k$ is the usual lattice momentum. All states with energies in the interval $-2 t<\varepsilon<2 t$ are extended due to the Bloch theorem. The states of energies $\varepsilon$ outside this interval are the gap states. These states are always present due to surfaces and impurities in real samples. ${ }^{10,15}$ Gap states are known to be exponentially localized. ${ }^{10,15}$ The energy dependence of the inverse of the localization length is $\lambda=\xi^{-1}=\ln \left[\left(\varepsilon+\sqrt{\varepsilon^{2}-4}\right) / 2\right]$. We will show how the decimation-renormalization method can be used to extract all of these known results.

Let the eigenstate, with eigenvalue $\varepsilon$, be $|n\rangle=\Sigma_{i} A_{i} c_{i}^{\dagger}|0\rangle$, where $|0\rangle$ is the vacuum state and $A_{i}$ is the amplitude of wave function $|n\rangle$ at site $i$. Then, after eliminating the odd sites, one has

$$
\varepsilon A_{2 i}=\left[\varepsilon t^{2} /\left(\varepsilon^{2}-2 t^{2}\right)\right]\left(A_{2 i+2}+A_{2 i-2}\right) .
$$

Thus we obtain the recursion relation

$$
t_{n}=\varepsilon t_{n-1}^{2} /\left(\varepsilon^{2}-2 t_{n-1}^{2}\right),
$$

with $t_{0}=t . t_{n}$ is the effective hopping coefficient of a particle from a site to a site of $2^{n}$ lattice constants away in the state of energy $\varepsilon$. Recursion relation (3) admits three fixed points $t^{*}=0, \varepsilon / 2$ and $-\varepsilon$. Figure 1 is the graphical demonstration of recursion relation (3). Several wellknown results can be obtained immediately from recursion relation (3).

If $t_{0}$ is between $A(=-|\varepsilon| / 2)$ and $B(=|\varepsilon| / 2)$ (dotted box in Fig. 1), i.e., when the energy is in the gap, $|\varepsilon|>2 t$, recursion relation (3) will lead $t_{n}$ to fixed point $t^{*}=0$ as shown by dotted line 1 in Fig. 1. According to Robbins and Koiller, ${ }^{12,14}$ the wave function will decay to zero asymptotically at infinity. For large $n, \mathrm{Eq}$. (3) has the asymptotic solution $t_{n} \sim \varepsilon \exp \left(-\lambda 2^{n}\right)$, with a positive constant $\lambda$ which is inversely proportional to the localization length. The exact expression of $\lambda$ in terms of energy $\varepsilon$ and the initial hopping coefficient $t$ is not easy to obtain, but it can be solved numerically. The inverse of the localization length can be derived from its exponential fitting. Figure 2 is the plot of $\ln (\lambda)$ vs $\ln (\varepsilon-2)$, which is the same as the exact result $\lambda=\xi^{-1}$ $=\ln \left[\left(\varepsilon+\sqrt{\varepsilon^{2}-4}\right) / 2\right]$. The asymptotic behavior of $t_{n}$ in-

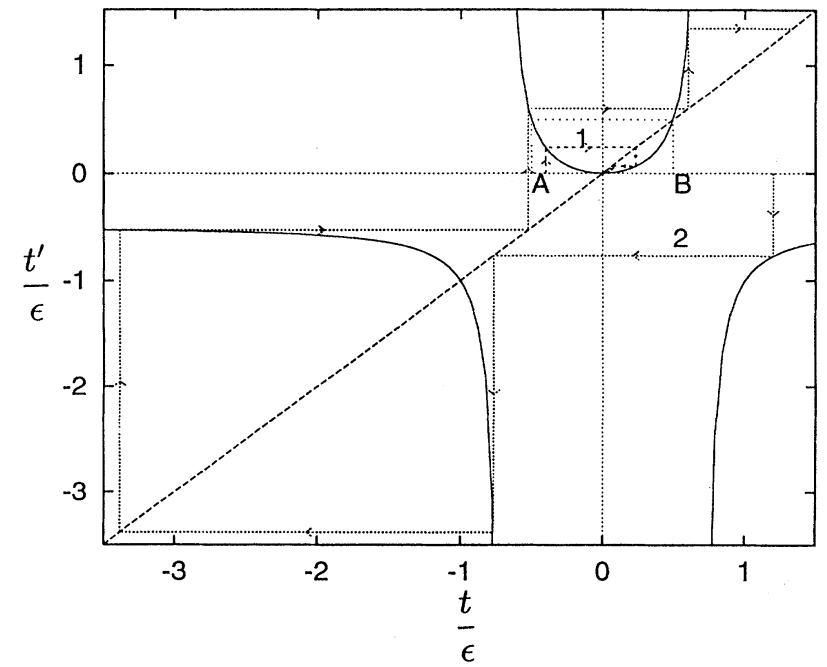

FIG. 1. Recursion relation curve and variant flow diagrams for the hopping coefficient in a one-dimensional lattice. The solid line is the recursion curve $t^{\prime}=\varepsilon t^{2} /\left(\varepsilon^{2}-2 t^{2}\right)$, and the dashed line is the curve of $t^{\prime}=t$. The dotted lines 1 and 2 are the flow diagrams of two different states.

dicates ${ }^{12,14}$ that the wave functions of such states at large distance $L\left(=2^{n}\right.$, after decimating $n$ times $)$ decay exponentially as $\Psi(L) \sim \exp (-\lambda L)$.

If $t_{0}$ starts from a point outside $A B$, i.e., when $-2 t \leq \varepsilon \leq 2 t$, then $t_{n}$ will always be larger than $|\varepsilon| / 2$ as shown by dotted line 2 in Fig. 1. In other words, the effective hopping coefficient between two sites is always nonzero no matter how far the two sites are separated. Therefore all states of energy $-2 t \leq \varepsilon \leq 2 t$ are delocalized (extended), ${ }^{12,14}$ as they should be according to the wellknown results. In particular, $\varepsilon=2 t$ (point $B$ ) is exactly at fixed point $t^{*}=\varepsilon / 2$, and the effective hopping coefficient corresponding to a state of this energy will be a constant. Obviously, a constant amplitude of the wave function at all sites is a solution in this case. This corresponds exactly to the state of $k=0$. On the other hand, the effective hopping coefficient of state $\varepsilon=-2 t$ (point $A$ ) will be constant after one iteration. Alternating amplitudes of a wave function on the lattice is a solution in this case. This is just the $k=\pi / a$ state in the known results.

Let us apply the same idea to the tight-binding model on the Sierpinski gasket (Fig. 3). Similar to the deriva-

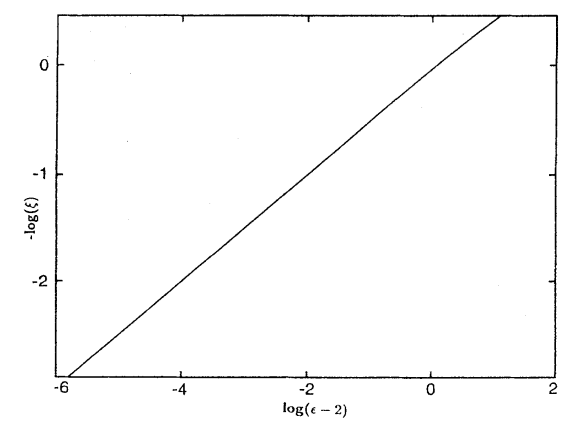

FIG. 2. $\ln (\lambda)$ vs $\ln (\varepsilon-2)$, where $\lambda(\varepsilon)$ is the inverse of localization length. The curve is exactly the same as $\lambda=\xi^{-1}=\ln \left[\left(\varepsilon+\sqrt{\varepsilon^{2}-4}\right) / 2\right]$. 


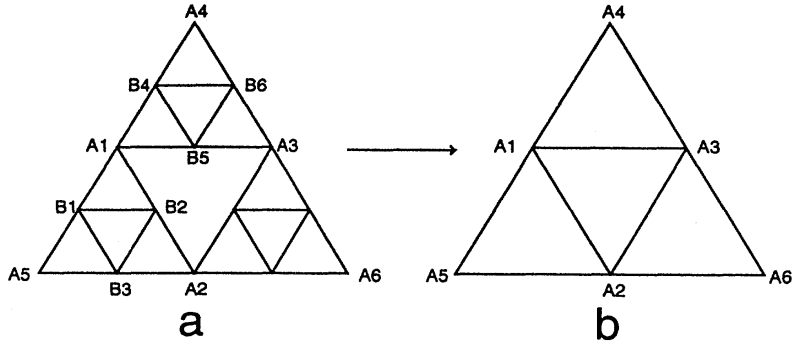

FIG. 3. Sierpinski gaskets before and after decimation. $A$ 's and $B$ 's are the amplitudes of the wave function at the corresponding sites.

tion of Eq. (2) for the 1D case, it is easy to show

$$
\begin{aligned}
& \varepsilon A_{1}=t\left(B_{1}+B_{2}+B_{4}+B_{5}\right), \\
& \varepsilon B_{1}=t\left(B_{2}+B_{3}+A_{1}+A_{5}\right), \\
& \varepsilon B_{2}=t\left(B_{1}+B_{3}+A_{1}+A_{2}\right), \\
& \varepsilon B_{3}=t\left(B_{1}+B_{2}+A_{2}+A_{5}\right), \\
& \varepsilon B_{4}=t\left(B_{5}+B_{6}+A_{1}+A_{4}\right), \\
& \varepsilon B_{5}=t\left(B_{4}+B_{6}+A_{1}+A_{3}\right), \\
& \varepsilon B_{6}=t\left(B_{4}+B_{5}+A_{3}+A_{4}\right),
\end{aligned}
$$

where $A$ 's and $B$ 's are the amplitudes of the wave function at the sites shown in Fig. 3. Using the decimation method, we will eliminate the $B$ 's in Eq. (4) and derive a equation which contains only $A$ 's. After some simple algebra, we have

$$
\varepsilon A_{1}=\frac{t^{2}}{\varepsilon-3 t}\left(A_{2}+A_{3}+A_{4}+A_{5}\right) .
$$

Therefore the recursion relation is

$$
t_{1}=t^{2} /(\varepsilon-3 t) \text {. }
$$

$t_{1}$ is, therefore, the effective hopping coefficient of a particle between two nearest $A$ sites in a state of energy $\varepsilon$. Recursion relation (6) has the two fixed points $t^{*}=0$ and $\varepsilon / 4$. Figure 4 is a graphical demonstration of recursion relation (6). The localization properties are determined by these fixed points.

The fixed point $t^{*}=\varepsilon / 4$ characterizes the behavior of extended states because of the nonzero coupling coefficient. $^{12}$ Therefore all of those states which will flow to this fixed point are delocalized. For example, the effective hopping coefficient of a state of energy $\varepsilon=4 t$ will be a constant under recursion relation (6). It is easy to show that constant amplitudes of a wave function on all sites is a solution. In order to obtain the set of the delocalized states, we shall use recursion relation (6) by going backwards. Solving

$$
\varepsilon / 4=t^{2} /(\varepsilon-3 t),
$$

for $t$, we have $t=\varepsilon / 4$ and $t=-\varepsilon . t=\varepsilon / 4$ describes the state of energy $\varepsilon=4 t$, which we mentioned before. The second solution gives us another extended state of energy

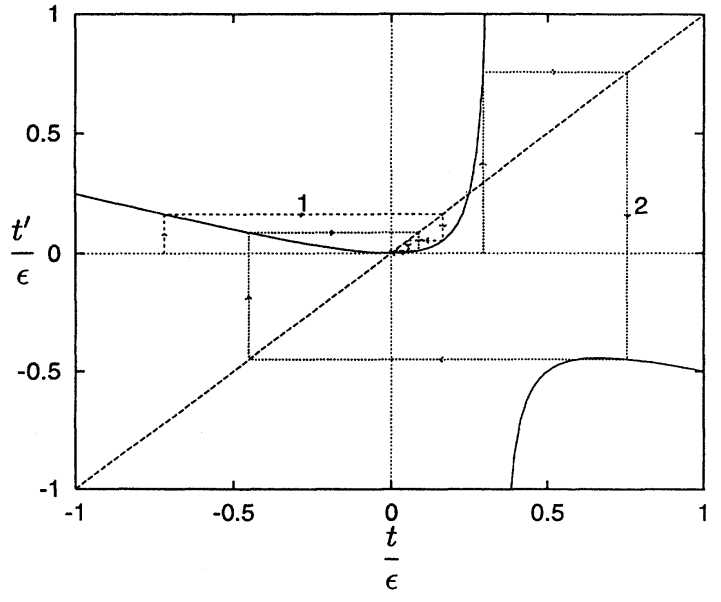

FIG. 4. Recursion relation curve and variant flow diagrams for the hopping coefficient on the Sierpinski gasket. The solid line is the recursion curve $t^{\prime}=t^{2} /(\varepsilon-3 t)$, and the dashed line is the curve of $t^{\prime}=t$. The flow diagrams 1 and 2 (dotted line) correspond to two different states.

$\varepsilon=-t$. According to recursion relation (6), the effective hopping coefficient of a particle in this state will be a constant value of fixed point $t^{*}=\varepsilon / 4$ after one decimation. Thus the amplitudes of the wave function on those $A$ sites (Fig. 3) are the same (but those on $B$ sites may not be the same); i.e., the wave function has an alternating spatial structure on the Sierpinski gasket. Substituting $t_{1}$ by $-\varepsilon$ in recursion relation (6), we have

$$
-\varepsilon=t^{2} /(\varepsilon-3 t) \text {. }
$$

There are two roots $t=(3 \pm \sqrt{5}) \varepsilon / 2$, which correspond to another two extended states of energies $\varepsilon=(3 \mp \sqrt{5}) \varepsilon / 2$. Since the effective hopping coefficients of a particle in the two states will take a constant value of $t^{*}=\varepsilon / 4$ after two decimations, the amplitudes of their wave function are the same on those sites of the Sierpinski gasket which are constructed from a large length scale of $4 a$. All other extended states can be obtained and characterized by continuing the process. These isolated extended states correspond to those minimum points in Figs. 5(a) and 5(b). The self-similar nature of this set is clearly noted.

It can be shown that, for energy $\varepsilon$ not in this set of extended states, recursion relation (6) will lead $t_{n}$ to the fixed point $t^{*}=0$. Thus the wave function will decay to zero asymptotically at infinity. ${ }^{12,14}$ Let us distinguish the following cases. (1) For large $n$ and $\varepsilon \neq 0$ or $3 t$, the difference equation (6) becomes approximately $t_{n} \approx t_{n-1}^{2} / \varepsilon$. The asymptotic solution of $t_{n}$ is $t_{n}$ $\sim \varepsilon \exp \left(-\lambda 2^{n}\right)$. Thus the wave functions decay exponentially as $\Psi(L) \sim \exp (-\lambda L) .^{12,14}$ (2) for $\varepsilon=0$ or $3 t$, the solution of the different equation (6) is $t_{n}=(-3)^{-n} t$. Therefore the state of $\varepsilon=0$ decays to zero at large distance in a power-law fashion as $\Psi(L) \sim L^{-p}$, with $p=\ln (3) / \ln (2) \simeq 1.58$ since $L=2^{n} .^{12,14}$ States with such behavior are called critical states ${ }^{8,10-12}$ which are somewhere in between extended and exponentially localized. It is interesting to note that $p$ is exactly the fractal dimension of the Sierpinski gasket. This result may suggest 

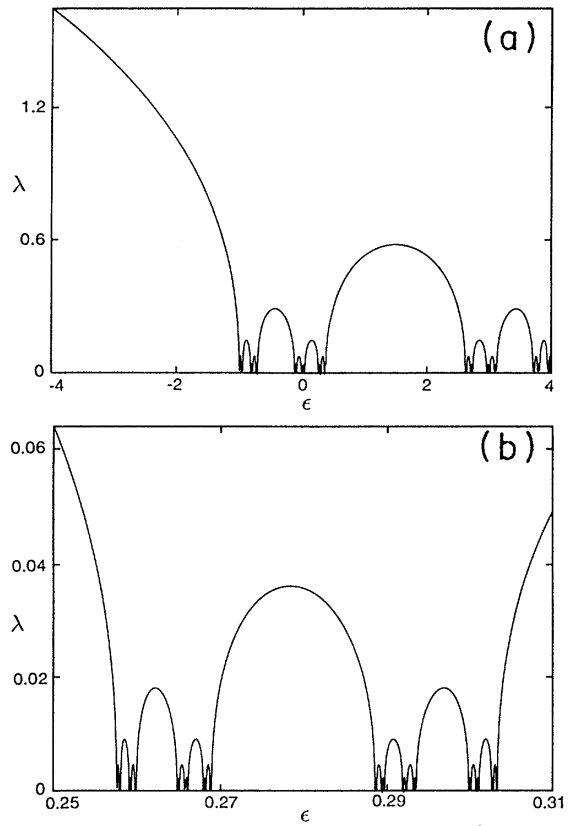

FIG. 5. $\lambda(\varepsilon)$, the inverse of localization length, in two different energy ranges.

that the support of the functions is the whole Sierpinski gasket. It is straightforward to find numerically the $t_{n}(\varepsilon)$ from recursion relation (6). Therefore $\lambda(\varepsilon)$ can be obtained by fitting $t_{n}$ to $\exp \left(-\lambda 2^{n}\right)$. Figures $5(\mathrm{a})$ and $5(\mathrm{~b})$ are the plots of $\lambda(\varepsilon)$ in different energy ranges. Again, they reveal a self-similar pattern.

In conclusion, we have presented a case where there exist localized, critical, and extended states. A method is proposed to study the electronic structure, namely, using the decimation-renormalization technique to derive the recursion relation for the hopping coefficient, from which one can obtain the asymptotic behavior of a wave function. It is interesting to note that the mathematical method used by Domany et al. ${ }^{13}$ is similar to the one proposed here. However, the physics is quite different. Domany et al. studied the recursion relation of the dimensionless quantity $\varepsilon / t$ instead of $t / \varepsilon$. Thus Domany et al. investigated how the energy splits with an increase of system size, and it is natural for them to obtain the density of states. On the other hand, we look at how a given energy wave function is connected at two different sites. This gives us a new way of looking at the localization problem. The method has been used to reexamine the well-known one-dimensional tight-binding model, and the known results have been reproduced. The method has also been applied to the tight-binding model on the Sierpinski gasket, with surprising results. Contrary to the common wisdom that all states are localized on the Sierpinski gasket, ${ }^{13}$ we have shown that there exists an infinite number of isolated extended states in the Sierpinski gasket. The set of these extended states forms a Cantor set which is self-similar. The rest of the states are exponentially localized except two states which are localized in a power-law fashion. For the two power-lawlocalized states, their exponents are found to be equal to the fractal dimension of the Sierpinski gasket. Furthermore, it has been shown that exponential localization lengths of states on the Sierpinski gasket reveal a selfsimilar pattern. Although the above results are obtained from the analysis of the tight-binding model, one should expect the conclusions to apply to any noninteraction particle systems on the Sierpinski gasket from universality arguments. However, interacting particle systems may behave differently. The method and idea are quite general. It can be used to solve other models and in other spaces, especially in those finitely ramified fractal spaces. It will be interesting to apply this method to similar problems in fractal spaces of different fractal dimensions. The results from such studies may help us understand how localization properties change with spatial dimensions. It will also be interesting to see whether the method can be used to analyze 1D quasiperiodic systems such as $\mathrm{Fi}$ bonacci lattices. Previous results in such systems are obtained either from numerical approaches ${ }^{11}$ or based on perturbative procedures. ${ }^{10}$ Exact results may be expected if the method can be applied to such problems. The detailed structure of the set of an infinite number of extended states deserves a more detailed study.

The author thanks Dr. Z. Q. Zhang, Professor C. Campbell, Professor S. Feng, Dr. M. K. Y. Wong, and Dr. T. K. Ng for stimulating discussions. The author also acknowledges Professor Nelson Cue for his useful comments. This work was supported by UPGC, Hong Kong, through a RGC Grant.
${ }^{1}$ D. Schechtman, I. Blech, D. Gratias, and J. W. Cahn, Phys. Rev. Lett. 53, 1951 (1984).

${ }^{2}$ P. W. Anderson, Phys. Rev. 109, 1492 (1958).

${ }^{3}$ D. J. Thouless, Phys. Rep. 13, 93 (1974); Phys. Rev. Lett. 39, 1167 (1977); D. C. Licciardello and D. J. Thouless, J. Phys. C 8, 4157 (1975).

${ }^{4}$ E. Abrahams, P. W. Anderson, D. C. Licciardello, and T. V. Ramakrishnan, Phys. Rev. Lett. 42, 673 (1979).

${ }^{5}$ Localization, Interaction, and Transport Pheomena, edited by B. Kramer, G. Bergmann, and Y. Bruynseraede (SpringerVerlag, Berlin, 1985).

${ }^{6}$ R. Merlin, K. Bajema, R. Clarke, F. Y. Jung, and P. K. Bhattacharaya, Phys. Rev. Lett. 55, 1768 (1985); M. W. C. Dharmawardana, A. H. MacDonald, D. J. Lockwood, J. M. Baribeau, and D. C. Houghton, ibid. 58, 1761 (1987).
${ }^{7}$ J. Todd, R. Merlin, R. Clarke, K. M. Mohanty, and J. D. Axe, Phys. Rev. Lett. 57, 1157 (1986); 59, 2237 (1987).

${ }^{8}$ J. B. Sokoloff and J. V. Jose, Phys. Rev. Lett. 49, 334 (1982).

${ }^{9}$ M. Kohmoto, L. P. Kadanoff, and C. Tang, Phys. Rev. Lett. 50, 1870 (1983).

${ }^{10}$ Q. Niu and F. Nori, Phys. Rev. Lett. 57, 2057 (1986).

${ }^{11}$ S. Das Sarma and X. C. Xie, Phys. Rev. B 37, 1097 (1988).

${ }^{12}$ R. B. Capaz, B. Koiller, and S. L. A. de Queiroz, Phys Rev. B 42, 6402 (1990).

${ }^{13}$ E. Domany, S. Alexander, D. Bensimon, and L. P. Kadanoff, Phys. Rev. B 28, 3110 (1983).

${ }^{14}$ M. O. Robbins and B. Koiller, Phys. Rev. B 32, 4576 (1985).

15J. M. Luck, Phys. Rev. B 39, 5834 (1989); K. W. K. Shung, L. M. Sander, and R. Merlin, Phys. Rev. Lett. 61, 455 (1988). 